\title{
EST and EST-SSR marker resources for Iris
} Shunxue Tang1, Rebecca A Okashah1, Marie-Michele Cordonnier-Pratt ${ }^{2}$, Lee H Pratt ${ }^{2}$, Virgil Ed Johnson ${ }^{2}$, Christopher A Taylor ${ }^{1}$, Michael L Arnold ${ }^{3}$
and Steven J Knapp*1

Address: ${ }^{1}$ Institute of Plant Breeding, Genetics, and Genomics, The University of Georgia, Athens, GA 30602, USA, ${ }^{2}$ Laboratory for Genomics and Bioinformatics, The University of Georgia, Athens, GA 30602, USA and ${ }^{3}$ Department of Genetics, The University of Georgia, Athens, GA 30602, USA

Email: Shunxue Tang - tangs@uga.edu; Rebecca A Okashah - rereoka@uga.edu; Marie-Michele Cordonnier-Pratt - mpratt@plantbio.uga.edu; Lee H Pratt - lpratt@plantbio.uga.edu; Virgil Ed Johnson - vjohnson@plantbio.uga.edu; Christopher A Taylor - taylor75@uga.edu; Michael L Arnold - arnold@plantbio.uga.edu; Steven J Knapp* - sjknapp@uga.edu

* Corresponding author

Published: 10 June 2009

BMC Plant Biology 2009, 9:72 doi:10.1 | 86/147|-2229-9-72
Received: 24 November 2008

Accepted: 10 June 2009

This article is available from: http://www.biomedcentral.com/147I-2229/9/72

(C) 2009 Tang et al; licensee BioMed Central Ltd.

This is an Open Access article distributed under the terms of the Creative Commons Attribution License (http://creativecommons.org/licenses/by/2.0), which permits unrestricted use, distribution, and reproduction in any medium, provided the original work is properly cited.

\begin{abstract}
Background: Limited DNA sequence and DNA marker resources have been developed for Iris (Iridaceae), a monocot genus of 200-300 species in the Asparagales, several of which are horticulturally important. We mined an I. brevicaulis-I. fulva EST database for simple sequence repeats (SSRs) and developed ortholog-specific EST-SSR markers for genetic mapping and other genotyping applications in Iris. Here, we describe the abundance and other characteristics of SSRs identified in the transcript assembly (EST database) and the cross-species utility and polymorphisms of $I$. brevicaulis-I. fulva EST-SSR markers among wild collected ecotypes and horticulturally important cultivars.
\end{abstract}

Results: Collectively, 6,530 ESTs were produced from normalized leaf and root cDNA libraries of I. brevicaulis (IB72) and I. fulva (IFI74), and assembled into 4,9I7 unigenes (I,066 contigs and 3,85 I singletons). We identified I,447 SSRs in I,I62 unigenes and developed 526 EST-SSR markers, each tracing a different unigene. Three-fourths of the EST-SSR markers (399/526) amplified alleles from IB72 and IFI74 and 84\% (335/399) were polymorphic between IB25 and IFI74, the parents of I. brevicaulis $\times I$. fulva mapping populations. Forty EST-SSR markers were screened for polymorphisms among 39 ecotypes or cultivars of seven species - 100\% amplified alleles from wild collected ecotypes of Louisiana Iris (I.brevicaulis, I.fulva, I. nelsonii, and I. hexagona), whereas $42-52 \%$ amplified alleles from cultivars of three horticulturally important species (I. pseudacorus, I. germanica, and $I$. sibirica). Ecotypes and cultivars were genetically diverse - the number of alleles/locus ranged from two to 18 and mean heterozygosity was 0.76 .

Conclusion: Nearly 400 ortholog-specific EST-SSR markers were developed for comparative genetic mapping and other genotyping applications in Iris, were highly polymorphic among ecotypes and cultivars, and have broad utility for genotyping applications within the genus. 


\section{Background}

Iris, a genus of 200-300 species in the Iridaceae (Asparagales), is one of the most widely admired and earliest cultivated garden flowers, having appeared in ancient Eygptian artifacts as early as 1950 B.C. [1]. The most widely cultivated, hybridized, and horticulturally important species are I.germanica (tall-bearded Iris), I.pseudacorus (yellow-flag Iris), and I.sibirica (Siberian Iris), each with numerous commercially important cultivars. Iris species are found in diverse habitats on every continent in the Northern Hemisphere and have been important models for the study of plant evolution, ecology, and hybrid speciation [2-8]. Chromosome numbers and ploidy are highly variable among and within species in the genus, ranging from $2 \mathrm{n}=16$ in I. attica to $2 \mathrm{n}=108$ in I. versicolor $[3,4]$. Similarly, haploid genome lengths are generally large and highly variable in the genus, ranging from 2,000 to $30,000 \mathrm{Mbp}$ [9].

Minimal genomic resources have been developed for Iris, a genus where forward genetic approaches have previously been applied to the study of life history and other traits by genotyping generic DNA markers, e.g., random amplified polymorphic DNA (RAPD) or retrotransposon display (IRRE) markers, in segregating populations developed from interspecific hybrids [7,9-14]. While such markers have facilitated linkage and quantitative trait locus (QTL) mapping in Iris, the uncertain orthology of RAPD and IRRE bands has precluded cross-referencing loci across populations and species. Simple sequence repeat (SSR), restriction fragment length polymorphism (RFLP), and single nucleotide polymorphism (SNP) markers are typically ortholog-specific and, consequently, have been widely used as DNA landmarks for synteny analysis and cross-referencing loci across populations [1517]. Thus far, a limited number of ortholog-specific DNA marker have been described for Iris [18]. The primary goal of the present study was to develop a sufficient number of ortholog-specific DNA markers for genome-wide comparative genetic mapping and other genotyping applications in I. brevicaulis $(\mathrm{x}=20)$, I. fulva $(\mathrm{x}=20)$, and other species in the genus by developing a small EST database and targeting SSRs in ESTs.

SSRs are ubiquitous in transcribed sequences, typically locus-specific and co-dominant, and often multi-allelic, highly polymorphic, and transferrable among species within genera [19-22]. EST databases have been a rich source of SSRs for the development of ortholog-specific EST-SSR markers for genotyping applications in numerous species of flowering plants [21-28]. When our study was initiated, a limited number of ESTs (201) had been deposited in GenBank http://www.ncbi.nlm.nih.gov/ Genbank/ for a single species in the genus, I. hollandica [29], and were insufficient for EST-SSR marker develop- ment. We developed a small EST database from cDNA sequences produced from normalized cDNA libraries of two species of Louisiana Iris (I. brevicaulis and I. fulva), partly to support the development of several hundred EST-SSR markers for comparative mapping and other genotyping applications in Louisiana Irises and partly to create DNA sequence and ortholog-specific DNA markers resources for the genus as a whole. Previous forward genetic analyses in I. brevicaulis and I. fulva identified QTL for several morphological, life history, and ecological traits [12,13,30-32]. Because ortholog-specific DNA markers were previously lacking for these genera, linkage groups and QTL identified in earlier analyses could not be cross-referenced and comparative genetic mapping was infeasible. Here, we describe the I. brevicaulis-I. fulva EST database and the development, cross-species utility, and polymorphisms of I. brevicaulis-I. fulva EST-SSR markers among wild collected ecotypes of four species of Louisiana Iris (I. brevicaulis, I. fulva, I. hexagona, and I. nelsonii) and horticulturally important cultivars of tall-bearded (I.germanica), yellow-flag (I.pseudacorus), and Siberian (I.sibirica) Iris.

\section{Results and Discussion \\ Development of a Leaf and Root EST Database for Iris}

Normalized leaf and root cDNA libraries were developed from I.brevicaulis (IB72) and I.fulva (IF174) ecotypes (root and leaf RNAs were pooled and a single cDNA library was constructed for each species). Library quality was checked by sequencing colony-PCR amplified inserts from 295 randomly selected cDNA clones split between the IB72 and IF174 libraries. Of the 295 clones, 251 (85.1\%) harbored inserts 800 bp or longer, three lacked inserts $(1.1 \%)$, and 290 (98.3\%) harbored unique inserts. Subsequently, 12,199 cDNA clones were single-passed sequenced and yielded 6,530 ESTs surpassing quality standards, 2,947 from the IB72 and 3,583 from the IF174 library. Less than $1 \%$ of the clones lacked cDNA inserts $(85 / 12,199)$. The vector- and quality-trimmed ESTs were deposited in GenBank (Acc. No. EX949962-EX956238 and FD387191-FD387443), annotated by BLASTX analyses against NCBI databases, assembled, and deposited in a database http://www.genome.uga.edu/IrisDB developed by modifying a previously described EST processing pipeline and database [33]. The mean length of vectorand quality-trimmed ESTs was $578.0 \mathrm{bp}$.

cDNA normalization minimized redundancy in the Iris EST database and yielded a wealth of unique cDNA sequences (unigenes) for EST-SSR marker development and other applications in Iris biology, breeding, and floriculture http://www.genome.uga.edu/IrisDB. The 6,530 ESTs assembled into 4,917 unigenes (3,851 singletons and 1,066 contigs); hence, $75.3 \%$ of the ESTs were unique and $78.3 \%$ of the unigenes were singletons. cDNAs were 
normalized using a protocol which has been applied in numerous plant and animal species and minimized abundant transcript resequencing [34-36]. cDNA populations in leaves are dominated by abundant transcripts, e.g., chlorophyl A/B binding proteins and rubisco, neither of which were abundant among transcripts isolated by sequencing normalized leaf cDNA libraries. The deepest contigs contained seven ESTs.

Unigenes ranged in length from 100 to $1,673 \mathrm{bp}$ with a mean length of $603.1 \mathrm{bp}$. Less than one-tenth of the unigenes $(433 / 4,917)$ were sequenced through the polyA tail. Mean GC contents, which were identical for I.brevicaulis $(45.4 \%)$ and I.fulva (45.3\%), were slightly greater than mean GC contents reported for onion (Allium cepa L.; 41.9\%) and Arabidopsis (42.7\%) transcripts [37,38]. Unigenes were annotated by BLASTX http:// www.ncbi.nlm.nih.gov/BLAST analyses against the NCBI Non-Redundant Protein http://www.ncbi.nlm.nih.gov/ RefSeq/ and UniProtKB Swiss-Prot and TrEMBL http:// www.expasy.ch/sprot/ databases. Using a BLASTX threshold of $\angle E=1^{10}$, significant similarities were found and putative functions were identified for 2,390 Iris unigenes $(48.6 \%)$. Thirty-two $(0.6 \%)$ additional unigenes were similar to genes of unknown function. Significant similarities were not found for the other 2,495 Iris unigenes $(50.8 \%)$. The fraction of unigenes homologous to cDNAs encoding known function genes was similar to onion, an economically important species in the Asparagales [38].

The Louisiana Iris ESTs developed in the present study have moderately increased DNA sequence resources for Iris, which were previously minimal http:// www.ncbi.nlm.nih.gov/, and supplied ESTs for an important basal species in the Asparagales, a family where DNA sequence information has primarily been produced for onion, asparagus (Asparagus officinalis $\mathrm{L}$.), and model species [38,39]. van Doorn et al. [29] previously described 201 I. hollandica ESTs from a tepal cDNA library. Other than the latter, 607 nucleotide sequences for 104 species of Iris had previously been deposited in public databases, the bulk of which were for a limited number of DNA sequence motifs commonly targeted in phylogenetic analyses, e.g., matK. The Sanger ESTs described here were produced before the emergence of next-generation DNA sequencing technologies, which have dramatically increased DNA sequencing throughput and are facilitating deeper and broader DNA sequencing than was previously practical in species with limited DNA sequence resources [40-42]. The Sanger ESTs we produced, while limited in number, build the foundation for deeper transcriptome sequencing in Iris using next-generation technologies.

\section{Abundance, Characteristics, and Distribution of SSRs in Louisiana Iris ESTs}

SSRs were highly frequent in the Louisiana Iris EST database (Figures 1，2; Additional File 1; http:// www.genome.uga.edu/IrisDB). We identified 1,447 perfect SSRs $(n \geq 5)$ in 1,162 unigenes. One-fourth of the 4,917 unigenes in the transcript assembly harbored at least one SSR, a frequency which was much greater than the frequency range $(2-12 \%)$ in many other flowering plants $[20,21,24,43]$. The mean SSR density was one per 2,048 bp, which was much higher than the density found in onion $(1 / 25 \mathrm{~kb}$; [38]), another species in the Asparagales, and Arabidopsis (1/14 kb; [44]). When the transcript assembly was mined for perfect and imperfect repeats, 3,487 SSRs $(n \geq 5)$ were identified in 2,037 unigenes $(41.4 \%)$ with a mean density of approximately one SSR per 850 bp; imperfect repeats are interrupted short tandem repeats.

SSR repeat numbers ranged from 5 to 30 and lengths ranged from 10 to $69 \mathrm{bp}$ (Figure 1; Additional File 1). Of the 1,447 perfect SSRs, $1,077(72.9 \%)$ were 14 bp or longer and $694(48.0 \%)$ were 18 bp or longer. The mean repeat number was 9 and the mean repeat length was 23 bp. Of the 1,447 perfect repeats, 807 were dinucleotides $(55.8 \%)$ and 569 were trinucleotides $(39.3 \%)$. The most common repeat motifs were AG/CT (50.1\%), AAG/CTT (18.9\%), and AGG/CCT (7.6\%) (Figure 2). Slightly more than two-thirds of the SSRs were located in UTRs $(61.4 \%$ in 5 '-UTRs, $8.3 \%$ in $3^{\prime}$-UTRs, and $30.3 \%$ in exons). Of repeats identified in UTRs, $62.3 \%$ were dinucleotides and $31.4 \%$ were trinucleotides. Conversely, of repeats identified in CDSs, $17.4 \%$ were dinucleotides and $82.6 \%$ were trinucleotides (Additional File 1). The low frequency of SSRs identified in 3'-UTRs was primarily a function of 5'-

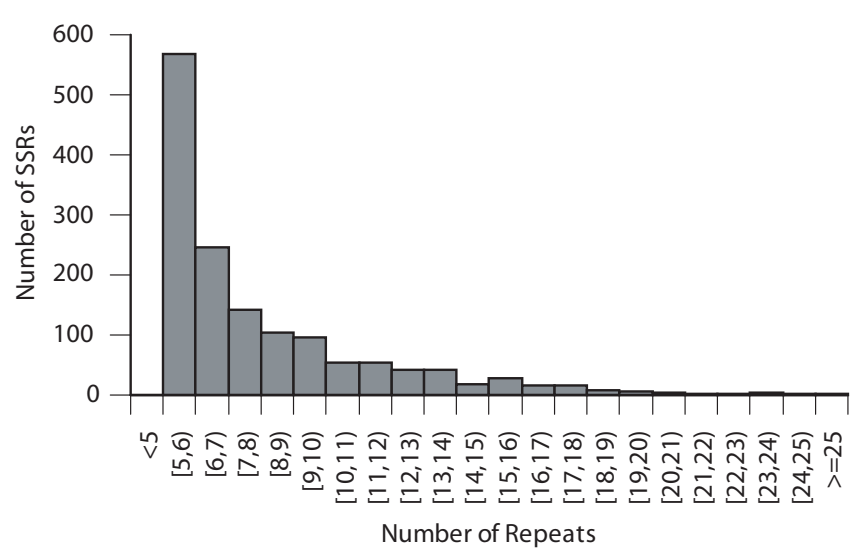

Figure I

Distribution of repeat counts for simple sequence repeats (SSRs) identified in I, 162 unigenes in the $I$. brevicaulis-I. fulva EST database. 


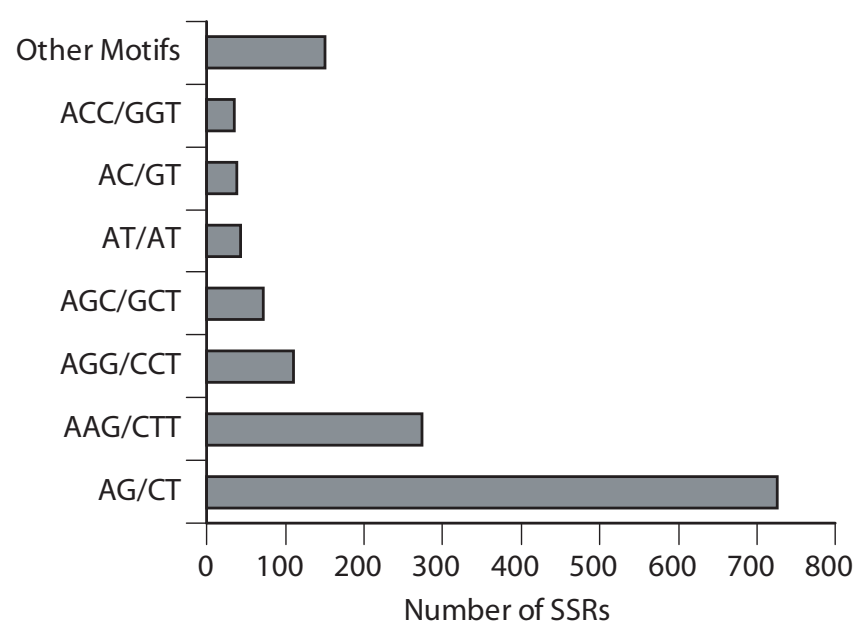

Figure 2

Distribution of dinucleotide and trinucleotide repeats identified in I, I62 unigenes in the $I$. brevicaulis-I. fulva EST database.

end sequencing, which yielded significantly fewer 3 ' than 5 '-UTR sequences $(8.8 \%$ of the unigenes harbored polyA tails).

The most common dinucleotide repeat motif was AG/CT, which constituted $89.8 \%$ of the dinucleotide repeats identifed in Iris ESTs and has been the most common dinucleotide repeat identified in other plant EST databases $[20,21,24,43]$. AG/CT repeats have been widely targeted for EST-SSR marker development in plants because, in addition to being highly abundant, they are often highly polymorphic, more abundant in UTRs than CDSs, seldom associated with transposons, and consistently amplify and yield robust SSR markers $[20,24]$. The frequencies of trinucleotide repeats in CDSs and dinucleotide repeats in UTRs appear to be similar in wheat and Iris ([26]; Additional File 1).

Trinucleotide repeats are typically more abundant than dinucleotide repeats in plants [21]; however, dinucleotide repeats $(56 \%)$ were more abundant than trinucleotide repeats (39\%) in Iris. Trinucleotide repeats (54-78\%) have been more abundant than dinucleotide repeats (17$40 \%$ ) in analyses of EST databases of several grass species $[22,27,43,45]$. Of the EST-SSRs identified in wheat (Triticum aestivuum L.), 70\% were trinucleotides and 30\% were dinucleotides [27]. AAG/CTT and AGG/CCT (67.5\%) were the most abundant trinucleotide repeats in Iris, whereas GCC/GGC appears to be the most abundant trinucleotide repeat motif in other plants [19,20,24,45]. SSRs were more abundant in UTRs than CDSs in Iris, whereas they are more abundant in CDSs than UTRs in other plant species $[21,24,43,46]$. SSR abundance in $3{ }^{\prime}$ -
UTRs of Iris ESTs may have been underestimated in the present study by $5^{\prime}$ directional sequencing of cDNAs, which artificially skews the distribution. SSRs appear to be equally abudant in 5'- and 3'-UTRs in other plant species $[20,25]$. If this pattern holds in Iris, the frequency of SSRs in UTRs could be as great as $80 \%$, which implies the frequency of SSRs in Iris ESTs could be greater than reported here (Additional File 1).

\section{Louisiana Iris EST-SSR Marker Development, Screening,} Allele Length Polymorphisms, and Cross-Species Utility SSRs with $n \geq 6$ repeats were selected from 526 unigenes for primer design and marker development (SSR primer sequences, allele lengths, repeat motifs, and other characteristics of the SSR markers are supplied in Additional File 2 ). The SSR markers were initially screened for amplication and allele length polymorphisms among three Lousiana Iris ecotypes (IB25, IB72, and IF174). Of the 526 ESTSSR markers, 399 (76\%) amplified alleles from at least one genotype (the null allele frequency was $2.7 \%$ ), whereas 127 (24\%) either failed to amplify alleles or produced amplicons which were too long (> $700 \mathrm{bp}$ ) or complex for genotyping. Of the 399 EST-SSR markers, 72 spanned introns longer than $200 \mathrm{bp}$ and amplified alleles longer than 700 bp and could not be genotyped (Additional File 2). Of the 327 SSR markers found to amplify alleles within the prescribed genotyping length range (100-700 bp), 283 (87\%) were polymorphic among IB25, IB72, and IF74. The number of polymorphic SSR markers/cross ranged from $247(76 \%)$ for IB25 $\times$ IB72 to $276(84 \%)$ for IB72 $\times$ IF174 (Additional File 2). Hence, most of the EST-SSR markers were polymorphic in I.brevicaulis $\times$ I.fulva mapping populations.

Forty I.brevicaulis-I.fulva EST-SSR markers were selected for more in-depth screening and analysis, primarily to quantify polymorphisms and assess transferability and utility among a broader sample of ecotypes, cultivars, and species. The 40 EST-SSR markers, which have been genetically mapped in I.brevicaulis $\times$ I.fulva and are distributed across the genome (unpublished data), were screened for amplification and allele length polymorphisms among 26 wild collected ecotypes of four Lousiana Iris species (I. brevicaulis, I. fulva, I. hexagona, and I. nelsonii) and 13 cultivars of tall-bearded (I.germanica), yellow-flag (I.pseudacorus), and Siberian (I.sibirica) Iris (Tables 1, 2; Additional File 3). Whilst $100 \%$ of the EST-SSR markers amplified alleles from Louisiana Iris ecotypes, half or slightly less than half amplified alleles from yellow-flag (52.5\%), Siberian (45.0\%), and tall-bearded (42.5\%) Iris cultivars. Of the 40 EST-SSR markers, only nine amplified alleles across the 39 ecotypes and cultivars (Figure 3; Additional File 4). One to three alleles/marker were amplified from triploid I.germanica, whereas one to two alleles/marker were amplified from the diploid species (Additional File 4). 


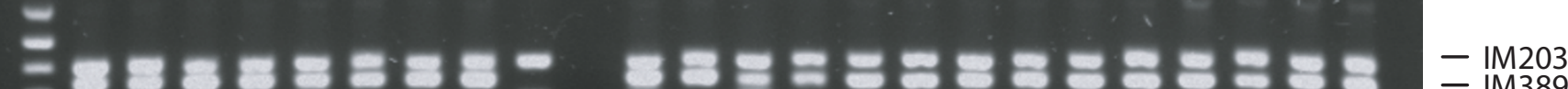

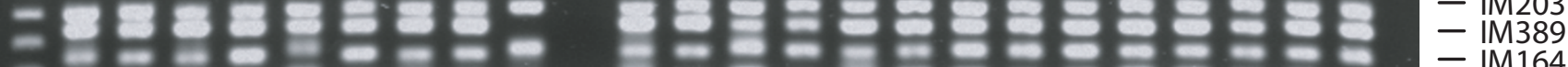 Ellotes 8

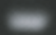

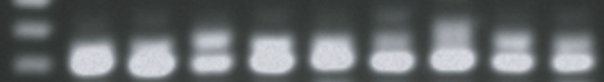

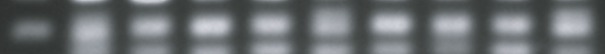

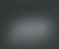

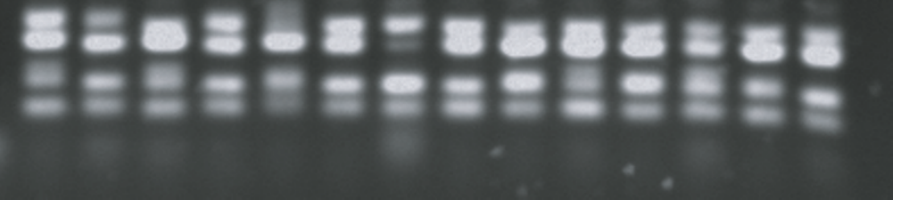 \\ $-\mathrm{IM} 27$ \\ - IM93 \\ - IM235 \\ - IM200}

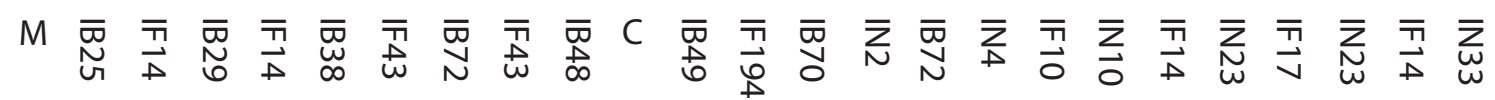
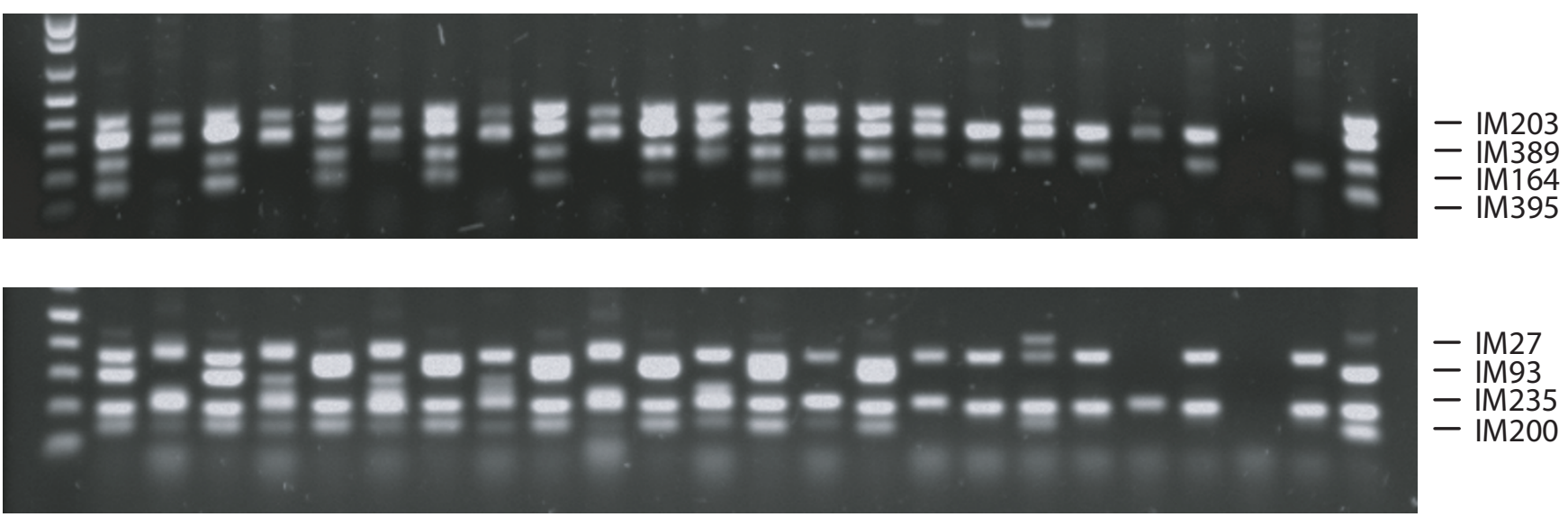

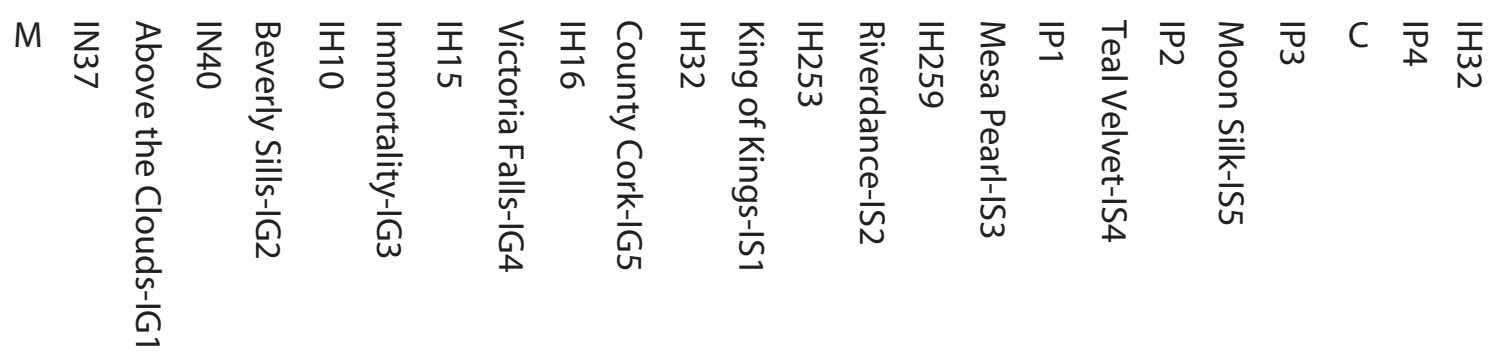

Figure 3

Genotypes for two multiplexes of four EST-SSR markers each screened for amplification and length polymorphisms on agarose among 39 ecotypes or cultivars of I. brevicaulis (IB), I. fulva (IF), I. nelsonii (IN), I. hexagona (IH), I. pseudacorus (IP), I. germanica (IG), and I. sibirica (IS). EST-SSR markers in the multiplexes were IM203, IM389, IMI64, and IM395 or IM27, IM93, IM235, and IM200. 
Table I: Linkage group (LG) assignment, number of alleles ( $n$ ), and mean heterozygosity $(h)$ estimated from genotypes of 40 EST-SSR markers among seven I. brevicaulis $\left(n_{B}\right.$ and $\left.h_{B}\right)$, six $I$. fulva $\left(n_{F}\right.$ and $\left.h_{F}\right)$, six I. hexagona $\left(n_{H}\right.$ and $\left.h_{H}\right)$, and seven $I$. nelsonii $\left(n_{N}\right.$ and $\left.h_{N}\right)$ ecotypes and among 26 Louisiana Iris ecotypes $\left(n_{L}\right.$ and $\left.h_{L}\right)$.

\begin{tabular}{|c|c|c|c|c|c|c|c|c|c|c|c|c|c|}
\hline $\begin{array}{l}\text { SSR } \\
\text { Marker }\end{array}$ & Motif & SSR Location & LG & $n_{B}$ & $n_{F}$ & $n_{H}$ & $n_{N}$ & $n_{L}$ & $h_{B}$ & $h_{F}$ & $h_{H}$ & $h_{N}$ & $h_{L}$ \\
\hline IM27 & $(A A G) \mid 2$ & 5' UTR & 18 & 2 & 1 & I & I & 4 & 0.24 & 0.00 & 0.00 & 0.00 & 0.64 \\
\hline IM55 & $(\mathrm{GAA}) 9$ & CDS & 1 & 6 & 7 & 4 & I & 10 & 0.76 & 0.83 & 0.68 & 0.00 & 0.82 \\
\hline IM6I & $(A G C) 7$ & CDS & 4 & 5 & 3 & 2 & 3 & 8 & 0.71 & 0.40 & 0.49 & 0.36 & 0.69 \\
\hline IM93 & $(\mathrm{ACC}) 8$ & CDS & 9 & 6 & 2 & 2 & 2 & 6 & 0.76 & 0.38 & 0.28 & 0.13 & 0.72 \\
\hline IM95 & $(\mathrm{AAG}) 8$ & 5' UTR & 17 & 6 & 1 & 3 & 3 & 11 & 0.80 & 0.00 & 0.50 & 0.65 & 0.82 \\
\hline IMI23 & (TCT) 10 & CDS & 3 & 7 & 4 & 2 & 5 & 8 & 0.83 & 0.65 & 0.50 & 0.61 & 0.81 \\
\hline IMI44 & $(\mathrm{CAG}) 6$ & CDS & 3 & 5 & 1 & 2 & 2 & 5 & 0.67 & 0.00 & 0.49 & 0.41 & 0.64 \\
\hline IMI46 & $(A C G) 6$ & CDS & 5 & 4 & 2 & 2 & 4 & 6 & 0.72 & 0.15 & 0.38 & 0.53 & 0.77 \\
\hline IMI52 & (CTA)6 & CDS & 6 & 4 & 3 & 2 & 2 & 5 & 0.37 & 0.57 & 0.28 & 0.34 & 0.64 \\
\hline IMI56 & (GAG)7 & CDS & 4 & 4 & 3 & 2 & I & 6 & 0.62 & 0.29 & 0.15 & 0.00 & 0.36 \\
\hline IMI 64 & (AGC)6 & CDS & 12 & 2 & 2 & 3 & 4 & 7 & 0.46 & 0.44 & 0.57 & 0.66 & 0.82 \\
\hline IMI65 & $(\mathrm{CAG}) 6$ & 3' UTR & 5 & 3 & 1 & 3 & 2 & 5 & 0.64 & 0.00 & 0.57 & 0.24 & 0.72 \\
\hline IMI96 & $(\mathrm{AAG}) 7$ & CDS & 10 & 6 & 5 & 3 & 7 & 16 & 0.68 & 0.72 & 0.54 & 0.83 & 0.90 \\
\hline IM200 & (GAA)23 & CDS & 1 & 9 & 4 & 2 & 3 & 10 & 0.87 & 0.51 & 0.28 & 0.44 & 0.77 \\
\hline IM203 & (CTT)7 & 5' UTR & 13 & 7 & 2 & 3 & 3 & 9 & 0.80 & 0.50 & 0.40 & 0.60 & 0.75 \\
\hline IM234 & (GCA)7 & CDS & 2 & 3 & 1 & 4 & 5 & 8 & 0.50 & 0.00 & 0.71 & 0.67 & 0.70 \\
\hline IM235 & (TGC)6 & 3' UTR & 12 & 4 & 2 & 4 & 3 & 10 & 0.66 & 0.44 & 0.58 & 0.62 & 0.83 \\
\hline IM283 & (CCT)8 & CDS & 21 & 2 & 2 & 2 & I & 4 & 0.13 & 0.15 & 0.15 & 0.00 & 0.55 \\
\hline IM299 & (TA) 6 & 3' UTR & 19 & 4 & 4 & 3 & 4 & 7 & 0.66 & 0.51 & 0.57 & 0.73 & 0.80 \\
\hline IM303 & $(\mathrm{TCC}) 6$ & 5' UTR & 16 & 6 & 5 & 2 & I & 10 & 0.80 & 0.69 & 0.15 & 0.00 & 0.77 \\
\hline IM319 & (GAG)7 & 5' UTR & 5 & 9 & 1 & 3 & 3 & 11 & 0.87 & 0.00 & 0.29 & 0.52 & 0.82 \\
\hline IM327 & $(\mathrm{GA}) 12$ & 5' UTR & 20 & 6 & 7 & 4 & 4 & 13 & 0.78 & 0.79 & 0.60 & 0.70 & 0.88 \\
\hline IM34I & (GAG)6 & 5' UTR & 12 & 3 & 1 & 3 & I & 7 & 0.50 & 0.00 & 0.65 & 0.00 & 0.69 \\
\hline IM348 & $(\mathrm{AGG}) 7$ & CDS & 2 & 7 & 3 & 8 & 4 & 11 & 0.70 & 0.49 & 0.85 & 0.46 & 0.77 \\
\hline IM364 & (CTT)6 & CDS & 9 & 9 & 4 & 2 & 2 & 10 & 0.87 & 0.65 & 0.15 & 0.24 & 0.73 \\
\hline IM377 & (ATT)6 & 5' UTR & 7 & 3 & 2 & 1 & 2 & 7 & 0.26 & 0.44 & 0.00 & 0.41 & 0.78 \\
\hline IM378 & (TTC) 9 & 5' UTR & 6 & 3 & 3 & 2 & 2 & 6 & 0.50 & 0.57 & 0.15 & 0.50 & 0.78 \\
\hline IM389 & (СTT)8 & CDS & 14 & 8 & 3 & 1 & 4 & 9 & 0.84 & 0.29 & 0.00 & 0.66 & 0.70 \\
\hline IM39I & (TTC) 12 & 5' UTR & 15 & 6 & 7 & 2 & 5 & 12 & 0.69 & 0.83 & 0.15 & 0.73 & 0.87 \\
\hline IM395 & (TC) 8 & 3' UTR & 15 & 3 & 3 & 3 & 4 & 7 & 0.57 & 0.61 & 0.57 & 0.53 & 0.80 \\
\hline IM402 & (СTT)। 10 & 5' UTR & 13 & 7 & 2 & 2 & 3 & 9 & 0.82 & 0.49 & 0.15 & 0.60 & 0.78 \\
\hline IM426 & (TCT)7 & 5' UTR & 4 & 6 & 4 & 2 & 4 & 11 & 0.76 & 0.71 & 0.38 & 0.62 & 0.85 \\
\hline IM429 & $(A G A) 8$ & 5' UTR & 14 & 3 & 3 & 3 & 2 & 8 & 0.44 & 0.40 & 0.40 & 0.34 & 0.75 \\
\hline IM442 & (GA)7 & 5' UTR & 16 & 2 & 1 & 1 & 2 & 2 & 0.34 & 0.00 & 0.00 & 0.46 & 0.47 \\
\hline IM450 & $(\mathrm{CAG}) 7$ & CDS & 2 & 7 & 2 & 5 & 4 & 10 & 0.74 & 0.28 & 0.53 & 0.64 & 0.70 \\
\hline IM460 & $(\mathrm{GA}) 7$ & 5' UTR & 8 & 7 & 2 & 3 & 4 & 13 & 0.83 & 0.28 & 0.54 & 0.66 & 0.85 \\
\hline IM486 & $(A G C) 7$ & CDS & 11 & 3 & 5 & 4 & 7 & 12 & 0.57 & 0.72 & 0.68 & 0.80 & 0.89 \\
\hline IM50I & $(A G) / 3$ & 5' UTR & 7 & 6 & 3 & 5 & 4 & 14 & 0.76 & 0.40 & 0.72 & 0.61 & 0.88 \\
\hline IM503 & (CT) 12 & 5' UTR & 10 & 7 & 6 & 5 & 7 & 18 & 0.82 & 0.82 & 0.68 & 0.76 & 0.89 \\
\hline IM526 & $(C A C) 6$ & CDS & 11 & 6 & 3 & 5 & 3 & 9 & 0.73 & 0.40 & 0.76 & 0.52 & 0.83 \\
\hline Mean & & & & 5.2 & 3.0 & 2.9 & 3.2 & 8.9 & 0.65 & 0.41 & 0.41 & 0.47 & 0.76 \\
\hline
\end{tabular}

The nine EST-SSR markers were highly polymorphic among tall-bearded, yellow-flag, and Siberian Iris cultivars; heterozygosities ranged from 0.77 to 0.91 (Table 2). Even though I. pseudacorus and I. sibirica belong to the same section (Limniri) as Louisiana Iris [5], a significant decrease in allele amplification was observed in these species, and was comparable to the decrease observed in $I$. germanica, a species from section Iris. Nevertheless, many of the I.brevicaulis-I.fulva EST-SSR markers developed in the present study amplify alleles from other species and should have broad utility in the genus $[1,5]$.
The 40 EST-SSR markers were highly polymorphic among and consistently amplified alleles from Louisiana Iris ecotypes; the null allele frequency was $0.5 \%$ (Table 1; Figure 3; Additional File 4). The number of alleles/locus $(n)$ ranged from two to 18 , the mean number of alleles/locus (n) was 8.9, heterozygosities of individual SSR markers ranged from 0.36 to 0.90 , and the mean heterozygosity (h) was 0.76 . Eighty to $100 \%$ of the EST-SSR markers were polymorphic, $n$ ranged from 2.9 to 5.2, and $h$ ranged from 0.41 to 0.65 among Louisiana Iris ecotypes (Table 1 ; Additional File 4). The number of species-specific alleles 
Table 2: Number of alleles $(n)$ and heterozygosities $(h)$ estimated from genotypes of nine EST-SSR markers among 26 Louisiana Iris ecotypes ( $n_{L}$ and $\left.h_{L}\right), 13$ yellow-flag, Siberian, and tall-bearded Iris cultivars $\left(n_{C}\right.$ and $\left.h_{C}\right)$, and across the 39 ecotypes and cultivars $\left(n_{T}\right.$ and $\left.h_{T}\right)$.

\begin{tabular}{|c|c|c|c|c|c|c|c|c|c|}
\hline $\begin{array}{l}\text { SSR } \\
\text { Marker }\end{array}$ & Motif & SSR Location & LG & $n_{L}$ & $n_{c}$ & $n_{T}$ & $h_{L}$ & $h_{c}$ & $h_{T}$ \\
\hline IM6I & $(A G C) 7$ & CDS & 4 & 8 & 10 & 15 & 0.69 & 0.82 & 0.88 \\
\hline IM93 & $(\mathrm{ACC}) 8$ & CDS & 9 & 6 & 6 & 8 & 0.72 & 0.77 & 0.79 \\
\hline IMI23 & (TCT) 10 & CDS & 3 & 8 & 6 & 14 & 0.81 & 0.82 & 0.91 \\
\hline IMI64 & $(\mathrm{AGC}) 6$ & CDS & 12 & 7 & 7 & 13 & 0.82 & 0.79 & 0.88 \\
\hline IMI96 & $(\mathrm{AAG}) 7$ & CDS & 10 & 16 & 10 & 20 & 0.90 & 0.87 & 0.93 \\
\hline IM200 & (GAA)23 & CDS & I & 10 & 8 & 18 & 0.77 & 0.83 & 0.90 \\
\hline IM327 & $(G A) 12$ & 5' UTR & 20 & 13 & 15 & 27 & 0.88 & 0.91 & 0.94 \\
\hline IM348 & $(A G G) 7$ & CDS & 2 & 11 & 8 & 15 & 0.77 & 0.86 & 0.88 \\
\hline IM39I & (TTC) 12 & 5' UTR & 15 & 12 & 9 & 17 & 0.87 & 0.87 & 0.93 \\
\hline Mean & & & & 10.1 & 8.8 & 16.3 & 0.80 & 0.84 & 0.89 \\
\hline
\end{tabular}

ranged from 26 in I. nelsonii to 101 in I. brevicaulis. Dinucleotide repeats were slightly more polymorphic than trinucleotide repeats. The mean number of alleles was $n=$ 10.6 for dinucleotide and 8.5 for trinucleotide repeats and the mean heterozygosity was $h=0.80$ for dinucleotide and 0.75 for trinucleotide repeats. SSRs in coding sequences ( $h$ $=0.78$ ) were only slightly less polymorphic than SSRs in UTRs $(h=0.73)$ (Table 1 ; Additional File 1$)$.

\section{Genetic Diversity Among Wild Collected Ecotypes and Horticulturally Important Cultivars}

Because only nine of the 40 I. brevicaulis-I.fulva EST-SSR markers amplified alleles from horticultural cultivars of I.germanica, I.pseudacorus, and I.sibirica, genetic distances and dendrograms were separately estimated from genotypes of the nine EST-SSR markers among accessions of all seven species and of the 40 EST-SSR markers among ecotypes of the four Louisiana Iris species (Figure 4; Additional File 4). Genetic distances $(G)$ ranged from 0.25 to 0.93 among Louisiana Iris ecotypes. The longest genetic distances were interspecific $(G=0.93$ between IB70 and IF10, IH32 and IN33, and IB25 and IF17), whilst the shortest genetic distances were intraspecific $(G=0.25$ between IF14 and IF17 and IH10 and IH16). Ecotypes assembled into species-specific clusters which were separated by greater genetic distances than ecotypes within species-specific clusters (Figure 4; Additional File 5). Genetic diversity was significant and diffuse among ecotypes or cultivars within species. Only a few EST-SSR markers were needed to identify (distinguish) ecotypes and cultivars.

\section{Conclusion}

cDNA sequences, an EST database, and EST-SSR markers were developed for comparative mapping, forward genetics, and other genotyping applications in Iris. cDNA normalization minimized transcript redundancy and small scale EST sequencing $(6,530)$ yielded 4,917 unigenes for gene discovery and DNA marker development. Perfect SSRs were identified in one-fourth of the unigenes $(1,162 /$ $4,917)$ and EST-SSR markers were developed for nearly half of the latter $(526 / 1,162)$. Three-fourths of the primers designed and tested (399/526) amplified alleles from reference ecotypes of $I$. brevicaulis and I. fulva and yielded robust EST-SSR markers. When 40 of the EST-SSR markers were screened for amplification, genotyping utility, and polymorphisms across species, 100\% amplified alleles from I. brevicaulis, I. fulva, I. hexagona, and I. nelsonii ecotypes, whereas $42-50 \%$ amplified alleles from I.germanica, I.pseudacorus, and I.sibirica ecotypes and cultivars. Hence, a large percentage of the I. brevicaulis-I. fulva ESTSSR markers developed in the present study should amplify alleles from other species and have broad utility in the genus. Finally, significant allelic diversity was discovered among Louisiana, yellow-flag, Siberian, and tallbearded ecotypes and cultivars; $90 \%$ of the EST-SSR markers were polymorphic and supply a wealth of orthologspecific DNA markers for biological and horticultural research in Iris.

\section{Methods cDNA Library Construction}

Normalized cDNA libraries were constructed from RNAs isolated from leaves and roots of an I.brevicaulis ecotype (IB72) and an I.fulva ecotype (IF174) using the Creator SMART cDNA Library Construction Kit (Clontech, Mountain View, CA). Total RNAs were isolated using Trizol (Invitrogen, Carlsbad, CA). First-strand cDNAs were synthesized using SuperScript ${ }^{\mathrm{TM}}$ III Reverse Transcriptase (Invitrogen, Carlsbad, CA). Double-strand cDNAs were normalized by duplex-specific nuclease (DSN) purified from Kamchatka crab hepatopancreas using the TrimmerDirect cDNA Normalization Kit (Evrogen, Moscow, Russia). Normalized cDNAs were size-fractionated, and 


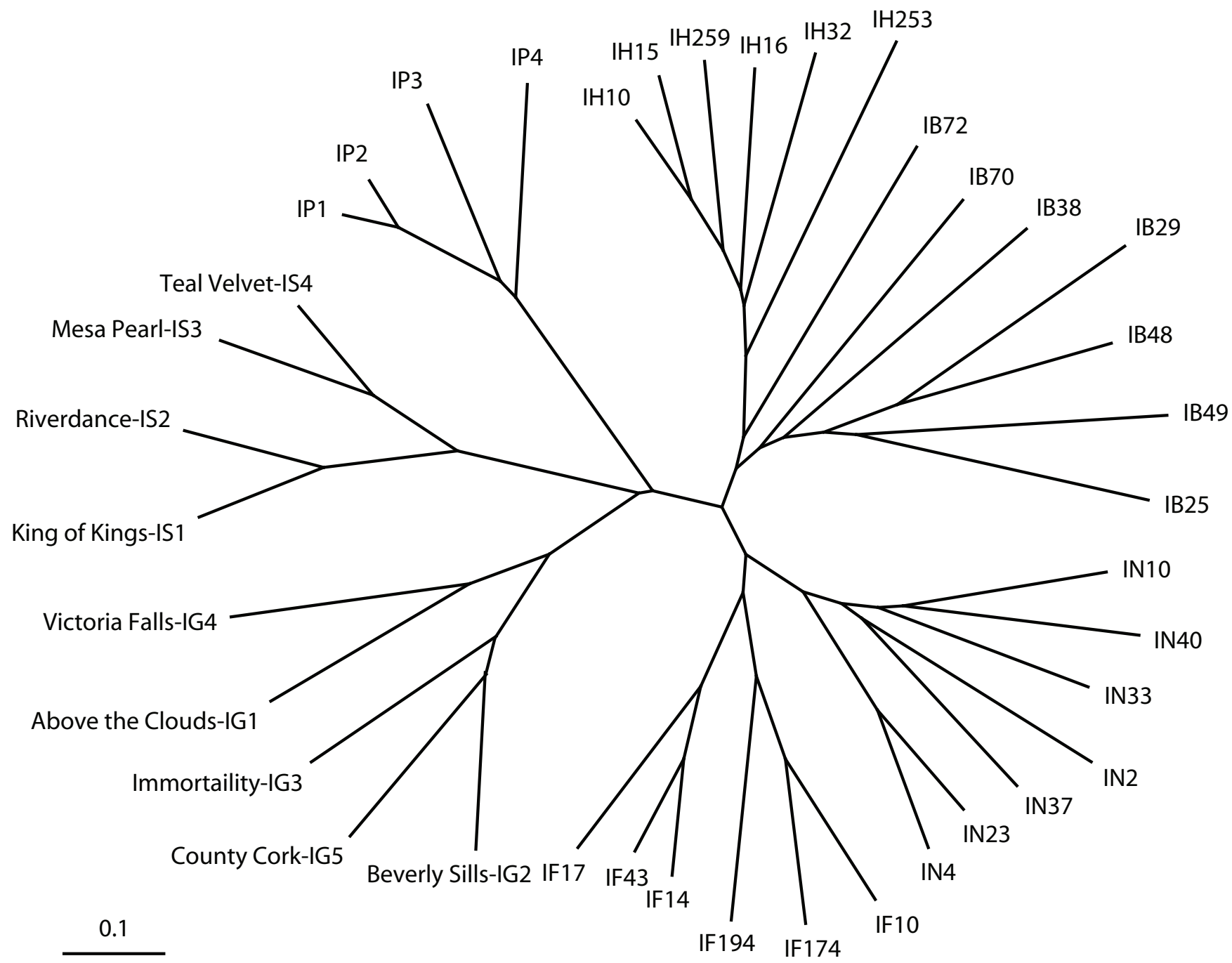

Figure 4

Dendrogram constructed from genetic distances estimated from genotypes of nine EST-SSR markers among seven I. brevicaulis (IB), six I. fulva (IF), six I. hexagona (IH), and seven I. nelsonii (IN) ecotypes and four I. pseudacorus (IP), five I. germanica (IG), and four I. sibirica (IS) cultivars.

cDNAs $>400$ bp were selected for cDNA library construction. Normalized and size selected cDNAs were digested with Sfi I (Fermentas, Glen Burnie, MD), directionally cloned into the vector pDNR-LIB, and electroporated into competent cell DH10B (Clontech, Mountain View, CA). To assess cDNA library quality and insert length distribution, inserts were amplified from 295 randomly selected cDNA clones by colony PCR.

\section{EST Database Development}

Collectively, 12,199 I. brevicaulis and I. fulva cDNA clones were 5 '-end single-pass Sanger sequenced at the Washington University Genome Sequencing Center, St. Louis, MO using M13 as the sequencing primer; roughly equal num- bers of randomly selected clones were sequenced from the two cDNA libraries. ESTs were processed, vector- and quality-trimmed, assembled, and annotated using a custom bioinformatics pipeline and deposited and displayed in Iris ESTdb http://www.genome.uga.edu/Iris, a relational EST database developed by modifying a previously described database [33]. Low-quality bases were PHREDtrimmed http://www.phrap.org/phredphrapconsed.html using a $\mathrm{Q}<16$ quality score (Q) threshold. Vector sequences were trimmed using Cross_Match http:// www.phrap.org/phredphrapconsed.html. cDNA sequences were screened for E. coli, chloroplast, and mitochondrial DNAs utilizing the SSAHA package http:// www.sanger.ac.uk/Software/analysis/SSAHA/. Vector- 
and quality-trimmed ESTs longer than 100 bp were assembled using MEGABLAST and CAP3 TGI Clustering Tools http://compbio.dfci.harvard.edu/tgi/software/. BLASTX http://www.ncbi.nlm.nih.gov/BLAST analyses were performed against the NCBI Non-Redundant Protein Database, UniprotSprot, and UniprotTrembl to identify putative functions of and annotate unique transcripts (unigenes).

\section{EST-SSR Discovery, Marker Development, and Length Polymorphism Screening}

Unigenes in the transcript assembly were screened for perfect repeat motifs using SSR-IT (http://www.gramene.org/

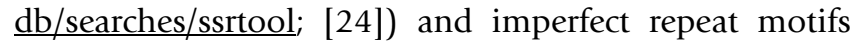
using FastPCR http://www.biocenter.helsinki.fi/bi/Pro grams/fastpcr.htm. SSRs with a minimum repeat count (n) threshold of $n \geq 5$ were selected for further analysis and EST-SSR marker development (Additional File 1). Flanking forward and reverse primers were designed for SSRs in 526 unigenes using Primer 3 (http:// frodo.wi.mit.edu/cgi-bin/primer3/primer3 www.cgi;

Additional File 2). To facilitate multiplex genotyping on an ABI3730 XL Capillary DNA Sequencer (Applied Biosystems, Foster City, CA), SSR primers were designed by uniformly varying target amplicon lengths from 100 to 450 bp and end-labeling forward primers with one of three fluorophores, 6FAM, HEX, or TAMRA (Additional File 2). The 526 SSR markers were screened for amplification and length polymorphisms among two I.brevicaulis ecotypes (IB72 and IB25) and an I.fulva ecotype (IF174) on agarose [47].

To assess cross-species amplification, transferability, and allele length polymorphisms, 40 of the 526 I. brevicaulis-I. fulva EST-SSR markers were screened among 26 ecotypes sampled from four Louisiana Iris species (I.brevicaulis, I.fulva, I. nelsonii, and I. hexagona), four yellow-flag Iris cultivars (I.pseudacorus), four Siberian Iris cultivars (I.sibirica), and five tall-bearded Iris cultivars (I.germanica) (Additional File 3). Louisiana Iris ecotypes were collected from Terrebonne Parish and St. Martinville Parish, Louisiana and I.pseudacorus ecotypes were collected from Spring Lake, San Marcos, TX. Tall-bearded and Siberian Iris cultivars were purchased from Schreiner Iris Gardens, Salem, Oregon. The 40 EST-SSR markers were previously mapped and distributed among 21 I.brevicaulis $\times$ I.fulva linkage groups (unpublished data). Genomic DNA was isolated from leaves of the 39 ecotypes or cultivars using a modified cetyltrimethylammonium bromide (CTAB) method [48]. SSR markers were genotyped on an ABI $3700 \mathrm{XL}$ Capillary DNA Sequencer as previously described $[47,49]$ and SSR allele lengths were ascertained using GeneMapper (Applied Biosystems, Foster City, CA). Heterozygosities $(H)$ of individual EST-SSR markers were estimated as described by Ott [50]. Genetic distances $(G)$ were esti- mated using the proportion of shared alleles estimator in Microsat, where $G=(1-p)$ and $p$ is the proportion of shared alleles http://hpgl.stanford.edu/projects/microsat/ . Neighbor-joining (NJ) trees were constructed using the NEIGHBOR program in PHYLIP http://evolution.genet ics.washington.edu/phylip.html and were drawn with TreeView http://taxonomy.zoology.gla.ac.uk/rod/ treeview.html.

\section{DNA Sequence Data}

Single-pass Sanger cDNA sequences (ESTs) for 6,530 I. brevicaulis and $I$. fulva clones have been deposited in GenBank (Acc. No. EX949962-EX956238 and FD387191FD387443).

\section{Authors' contributions}

ST developed the cDNA libraries, produced the ESTs, developed and screened the DNA markers, performed molecular and statistical genetic analyses, assisted with the development of the EST database and drafting the manuscript. RAO assisted with molecular analyses. MMCP, LHP, VEJ, and CAT designed and developed the EST database and performed bioinformatic analyses. MLA and SJK designed and coordinated the study and assisted with statistical analyses and drafting the manuscript.

\section{Additional material}

\section{Additional file 1}

Perfect simple sequence repeats $(1,447)$ identified in 1,162 unigenes in the Iris EST database.

Click here for file

[http://www.biomedcentral.com/content/supplementary/14712229-9-72-S1.xls]

\section{Additional file 2}

SSR motifs and repeat counts, unigene identifiers, primer sequences, and allele lengths for 526 Iris EST-SSR markers.

Click here for file

[http://www.biomedcentral.com/content/supplementary/14712229-9-72-S2.xls]

\section{Additional file 3}

Louisiana, yellow-flag, tall-bearded, and Siberian Iris ecotypes and cultivars screened for SSR allele length polymorphisms.

Click here for file

[http://www.biomedcentral.com/content/supplementary/1471-

2229-9-72-S3.xls]

\section{Additional file 4}

Allele length database for 40 EST-SSR markers among 39 ecotypes or cultivars of I. brevicaulis (IB), I. fulva (IF), I. nelsonii (IN), I. hexagona (IH), I. pseudacorus (IP), I. germanica (IG), and I. sibirica (IS).

Click here for file

[http://www.biomedcentral.com/content/supplementary/14712229-9-72-S4.xls] 


\section{Additional file 5}

Dendrogram constructed from genetic distances estimated from genotypes of 40 EST-SSR markers among seven I. brevicaulis (IB), six I. fulva (IF), six I. hexagona (IH), and seven I. nelsonii (IN) ecotypes. Click here for file

[http://www.biomedcentral.com/content/supplementary/14712229-9-72-S5.pdf]

\section{Acknowledgements}

This research was supported by grants from the National Science Foundation (DEB-0345 I 23) to M.L.A. and the Office of the Vice President for Research at the University of Georgia to S.J.K. and M.L.A.

\section{References}

I. Lyte C, Maynard P, Ellis JR, Service N, Rix M, Grey-Wilson C, Dickson-Cohen VC, Linnegar S, Bowley ME, Blanco-White A, Cohen O, Davis A, Jury S, Innes C, Christiansen H, Mathew B, Killens WR, Waddick JW, King C: A Guide to Species Irises: Their Identification and Cultivation. Cambridge, United Kingdom: Cambridge University Press; 1997.

2. Arnold ML, Bennett BD, Zimmer EA: Natural hybridization between Iris fulva and Iris hexagona: pattern of ribosomal DNA variation. Evolution 1990, 44:1512-152|.

3. Mitra J: Karyotype analysis of Bearded Iris. Botanical Gazette 1956, I 1 7:265-293.

4. Randolph LF, Mitra J, Nelson IS: Cytotaxonomic studies of Louisiana Irises. Botanical Gazette 1961, I 23:125-133.

5. Rodionenko Gl: The genus Iris L.: questions of morphology, biology, evolution, and systematics. London, United Kingdom: British Iris Society; 1987.

6. Arnold ML: Anderson's paradigm: Louisiana irises and the study of evolutionary phenomena. Mol Ecol 2000, 9(II):1687-1698.

7. Arnold ML: Iris nelsonii (Iridaceae): origin and genetic composition of a homoploid hybrid species. Am J Bot 1993, 80:577-583.

8. Makarevitch I, Golovnina K, Scherbik S, Blinov A: Phylogenetic relationships of the Siberian Iris species inferred from noncoding chloroplast DNA sequences. Intl Journal Plant Sci 2003, 164:229-237.

9. Kentner EK, Arnold ML, Wessler SR: Characterization of highcopy-number retrotransposons from the large genomes of the louisiana iris species and their use as molecular markers. Genetics 2003, 164(2):685-697.

10. Reeves G, Chase MW, Goldblatt P, Rudall P, Fay MF, Cox AV, Lejeune $B$, Souza-Chies T: Molecular systematics of Iridaceae: evidence from four plastid DNA regions. Am J Bot 200I, 88:2074-2087.

II. Arafeh RM, Sapir Y, Shmida A, Iraki N, Fragman O, Comes HP: Patterns of genetic and phenotypic variation in Iris haynei and $I$. atrofusca (Iris sect. Oncocyclus = the royal irises) along an ecogeographical gradient in Israel and the West Bank. Mol Ecol 2002, I I ( I):39-53.

12. Bouck A, Peeler R, Arnold ML, Wessler SR: Genetic mapping of species boundaries in Louisiana irises using IRRE retrotransposon display markers. Genetics 2005, I7I(3): 1289-1303.

13. Bouck A, Wessler SR, Arnold ML: QTL analysis of floral traits in Louisiana iris hybrids. Evolution 2007, 6 I (10):2308-2319.

14. Cornman RS, Arnold ML: Phylogeography of Iris missouriensis (Iridaceae) based on nuclear and chloroplast markers. Mol Ecol 2007, I6(2I):4585-4598.

15. Rafalski A: Applications of single nucleotide polymorphisms in crop genetics. Curr Opin Plant Biol 2002, 5(2):94-100.

16. Tanksley SD, Young ND, Paterson AH, Bonierbale MW: RFLP mapping in plant breeding: new tools for an old science. Biotechnology 1989, 7:257-264.

17. Taramino G, Tingey S: Simple sequence repeats for germplasm analysis and mapping in maize. Genome 1996, 39(2):277-287.

18. Burke JM, Arnold ML: Isolation and characterization of microsatellites in Iris. Molecular Ecology 1999, 8:1091-1092.
19. Power W, Machray GC, Povran J: Polymorphism revealed by simple sequence repeats. Trends Plant Sci 1996, I:215-222.

20. Morgante M, Hanafey M, Powell W: Microsatellites are preferentially associated with nonrepetitive DNA in plant genomes. Nat Genet 2002, 30(2): 194-200.

2I. Varshney RK, Graner A, Sorrells ME: Genic microsatellite markers in plants: features and applications. Trends Biotechnol 2005, 23(1):48-55.

22. Varshney RK, Sigmund R, Boerner A, Korzun V, Stein N, Sorrells ME, Langridge $P$, Graner A: Interspecific transferability and comparative mapping of barley EST-SSR markers in wheat, rye, and rice. Plant Sci 2005, 168:195-202.

23. Peakall R, Gilmore S, Keys W, Morgante M, Rafalski A: Cross-species amplification of soybean (Glycine max) simple sequence repeats (SSRs) within the genus and other legume genera: implications for the transferability of SSRs in plants. Mol Biol Evol 1998, I 5( I 0): I275- I 287.

24. Temnykh S, DeClerck G, Lukashova A, Lipovich L, Cartinhour S, McCouch S: Computational and experimental analysis of microsatellites in rice (Oryza sativa $L$.): frequency, length variation, transposon associations, and genetic marker potential. Genome Res 200I, I I(8): I44I-I452.

25. Thiel T, Michalek W, Varshney RK, Graner A: Exploiting EST databases for the development and characterization of genederived SSR-markers in barley (Hordeum vulgare L.). Theor Appl Genet 2003, I 06(3):4 I I-422.

26. Yu JK, Dake TM, Singh S, Benscher D, Li W, Gill B, Sorrells ME: Development and mapping of EST-derived simple sequence repeat markers for hexaploid wheat. Genome 2004, 47(5):805-818.

27. Yu JK, La Rota M, Kantety RV, Sorrells ME: EST derived SSR markers for comparative mapping in wheat and rice. Mol Genet Genomics 2004, 27 I(6):742-75I.

28. Heesacker A, Kishore VK, Gao W, Tang S, Kolkman JM, Gingle A Matvienko M, Kozik A, Michelmore RM, Lai Z, et al.: SSRs and INDELs mined from the sunflower EST database: abundance, polymorphisms, and cross-taxa utility. Theor Appl Genet 2008, I i 7(7): 102I-1029.

29. van Doorn WG, Balk PA, van Houwelingen AM, Hoeberichts FA, Hall RD, Vorst $O$, Schoot $C$ van der, van Wordragen MF: Gene expression during anthesis and senescence in Iris flowers. Plant Mol Biol 2003, 53:845-863.

30. Martin $\mathrm{NH}$, Bouck AC, Arnold ML: The genetic architecture of reproductive isolation in Louisiana irises: flowering phenology. Genetics 2007, I75(4):|803-|8|2.

31. Martin NH, Bouck AC, Arnold ML: Detecting adaptive trait introgression between Iris fulva and I. brevicaulis in highly selective field conditions. Genetics 2006, I 72(4):248I-2489.

32. Martin NH, Sapir Y, Arnold ML: The genetic architecture of reproductive isolation in Louisiana irises: pollination syndromes and pollinator preferences. Evolution 2008, 62(4):740-752.

33. Pratt LH, Liang C, Shah M, Sun F, Wang H, Reid SP, Gingle AR, Paterson $A H$, Wing R, Dean R, et al: Sorghum expressed sequence tags identify signature genes for drought, pathogenesis, and skotomorphogenesis from a milestone set of 16,801 unique transcripts. Plant Physiol 2005, 139(2):869-884.

34. Zhulidov PA, Bogdanova EA, Shcheglov AS, Vagner LL, Khaspekov GL, Kozhemyako VB, Matz MV, Meleshkevitch E, Moroz LL, Lukyanov SA, et al: Simple cDNA normalization using kamchatka crab duplex-specific nuclease. Nucleic Acids Res 2004, 32(3):e37.

35. Anisimova VE, Barsova EV, Bogdanova EA, Lukyanov SA, Shcheglov AS: Thermolabile duplex-specific nuclease. Biotechnol Lett 2009, 3 I (2):25I-257.

36. Bogdanova EA, Shagin DA, Lukyanov SA: Normalization of fulllength enriched cDNA. Mol Biosyst 2008, 4(3):205-2I2.

37. Analysis of the genome sequence of the flowering plant Arabidopsis thaliana. Nature 2000, 408(68 I 4):796-8I5.

38. Kuhl JC, Cheung F, Yuan Q, Martin W, Zewdie Y, McCallum J, Catanach $A$, Rutherford $P$, Sink KC, Jenderek $M$, et al.: $A$ unique set of $I I, 008$ onion expressed sequence tags reveals expressed sequence and genomic differences between the monocot orders Asparagales and Poales. Plant Cell 2004, I 6(I): I I4-I 25.

39. Kuhl JC, Havey MJ, Martin WJ, Cheung F, Yuan Q, Landherr L, Hu Y, Leebens-Mack J, Town CD, Sink KC: Comparative genomic analyses in Asparagus. Genome 2005, 48(6): 1052-1060. 
40. Metzker ML: Emerging technologies in DNA sequencing. Genome Res 2005, I5(12): 1767-1776.

41. Mardis ER: Next-generation DNA sequencing methods. Annu Rev Genomics Hum Genet 2008, 9:387-402.

42. Wheeler DA, Srinivasan M, Egholm M, Shen Y, Chen L, McGuire A, He W, Chen YJ, Makhijani V, Roth GT, et al.: The complete genome of an individual by massively parallel DNA sequencing. Nature 2008, 452(7/ 89):872-876.

43. Kantety RV, La Rota M, Matthews DE, Sorrells ME: Data mining for simple sequence repeats in expressed sequence tags from barley, maize, rice, sorghum and wheat. Plant Mol Biol 2002, 48(5-6):50I-5। 0 .

44. Cardle L, Ramsay L, Milbourne D, Macaulay M, Marshall D, Waugh R: Computational and experimental characterization of physically clustered simple sequence repeats in plants. Genetics 2000, I 56(2):847-854.

45. Asp T, Frei UK, Didion T, Nielsen KK, Lubberstedt T: Frequency, type, and distribution of EST-SSRs from three genotypes of Lolium perenne, and their conservation across orthologous sequences of Festuca arundinacea, Brachypodium distachyon, and Oryza sativa. BMC Plant Biol 2007, 7:36.

46. Metzgar D, Bytof J, Wills C: Selection against frameshift mutations limits microsatellite expansion in coding DNA. Genome Res 2000, I0(1):72-80.

47. Tang S, Kishore VK, Knapp SJ: PCR-multiplexes for a genomewide framework of simple sequence repeat marker loci in cultivated sunflower. Theor Appl Genet 2003, 107(1):6-19.

48. Murray MG, Thompson WF: Rapid isolation of high molecular weight plant DNA. Nucleic Acids Res 1980, 8(19):432I-4325.

49. Tang S, Yu JK, Slabaugh B, Shintani K, Knapp J: Simple sequence repeat map of the sunflower genome. Theor Appl Genet 2002, 105(8): I I 24-1 I 36.

50. Ott J: Analysis of human genetic linkage. Baltimore, Maryland: John Hopkins University Press; 1999.

Publish with Bio Med Central and every scientist can read your work free of charge

"BioMed Central will be the most significant development for disseminating the results of biomedical research in our lifetime. "

Sir Paul Nurse, Cancer Research UK

Your research papers will be:

- available free of charge to the entire biomedical community

- peer reviewed and published immediately upon acceptance

- cited in PubMed and archived on PubMed Central

- yours - you keep the copyright 